\title{
緭Irestice
}

\section{Contribuições das Tecnologias da Informação e Comunicação em Saúde como Estratégias de Enfrentamento às Zoonoses'}

\section{CONTRIBUTIONS OF INFORMATION AND COMMUNICATION TECHNOLOGIES IN HEALTH AS STRATEGIES FOR COPING ZOONOSIS}

Sebastião André Barbosa Junior ${ }^{1}$, Aderaldo Alexandrino de Freitas ${ }^{2}$ e Márcia Mello Costa De Liberal $^{3}$

\author{
1 Doutorando em Medicina Veterinária. Programa de \\ Pós-graduação em Medicina Veterinária da \\ Universidade Federal Rural de Pernambuco. \\ ORCID: https://orcid.org/0000-0002-5524-9204 \\ Email: sebastiaoandre.jr@hotmail.com \\ 2 Doutor em Ciência Veterinária. Departamento de \\ Medicina Veterinária da Universidade Federal Rural de \\ Pernambuco. \\ ORCID: https://orcid.org/0000-0002-8150-4897 \\ Email: aderaldo.doscariris@gmail.com \\ 3 Pós-doutora em Ciência Política. Escola Paulista de \\ Medicina, Universidade Federal de São Paulo. \\ ORCID: https://orcid.org/0000-0002-2589-1802 \\ Email: delibera|@uol.com.br
}

Correspondência: Rua Dom Manuel de Medeiros, s/n, Dois Irmãos, Recife - PE, Brasil. CEP: 52171-900.

Copyright: Esta obra está licenciada com uma Licença Creative Commons Atribuição-NãoComercial 4.0 Internacional.

Conflito de interesses: os autores declaram que não há conflito de interesses.

\section{Como citar este artigo}

Barbosa Júnior AS; Freitas AA de; Liberal MMC De. Contribuições das Tecnologias da Informação e Comunicação em Saúde como Estratégias de Enfrentamento às Zoonoses. Revista de Saúde Digital e Tecnologias Educacionais. [online], volume 5, n. 2. Editor responsável: Luiz Roberto de Oliveira. Fortaleza, julho de 2020, p. 14-29. Disponível em: http://periodicos.ufc.br/resdite/index. Acesso em "dia/mês/ano".

Data de recebimento do artigo: 20/11/2019

Data de aprovação do artigo: 30/04/2020

Data de publicação: 20/07/2020

\section{Resumo}

Objetivo: Sistematizar informações sobre as Tecnologias da Informação e Comunicação em Saúde que contribuam no enfrentamento das zoonoses. Método: Foram realizadas buscas nas bases de dados LILACS e SCIELO, com os descritores: "ANÁLISE ESPACIAL", "TELEMEDICINA" e "ZOONOSES", selecionando artigos científicos de pesquisas de origem no Brasil e dentro do período de 2014 a 2018. Resultados: Foram selecionados 12 artigos científicos, esses foram divididos em três categorias: Teleducação e Telemedicina, com dois; Sistemas de Informações em Saúde, com seis e Análise Espacial, com quatro. Na primeira categoria, destacaram-se experiências de teleducação, na segunda, o SINAN foi o SIS mais utilizado e, na Análise Espacial, destacaram-se estudos com os softwares TerraView, Google Earth e ArcGIS. Conclusão: A escassez de informações sobre as zoonoses foi ratificada

\footnotetext{
${ }^{1}$ Artigo desenvolvido a partir da monografia do curso de Especialização em Informática em Saúde do primeiro autor, realizado pela Universidade Federal de São Paulo (UNIFESP).
} 
com os poucos artigos científicos encontrados, mostrando dessa forma a demanda pela realização de atividades sobre zoonoses com subsídio das TICs no contexto do Sistema Único de Saúde.

Palavras-Chave: Doenças Infecciosas.

Zoonoses. Informática em Saúde. Sistema Único de Saúde.

\section{Abstract \\ Objective: To systematize information about Health Information and Communication Technologies that contribute to coping zoonosis. Method: Searches have performed in the LILACS and SCIELO databases with the descriptors: "SPACE ANALYSIS", "TELEMEDICINE" and "ZOONOSIS", selecting scientific articles from}

researches of origin in Brazil and from 2014 to 2018. Results: Twelve scientific articles have selected, divided into three categories: Teleducation and Telemedicine, with two, Health Information Systems with six and Spatial Analysis with four. In the first category, there were teleducation experiences, in the second, SINAN was the most used HIS and in Spatial Analysis were focused studies with TerraView, Google Earth and ArcGIS software. Conclusion: The scarcity of information on zoonosis has confirmed by the few scientific articles found, thus showing the demand for activities on zoonosis with ICT subsidy in the context of the Unified Health System.

Keywords: Health Informatics. Zoonosis. Infectious diseases. Unified Health System.

\section{Introdução}

A semântica da palavra zoonose vem do grego, Zoo, animal e gnosis, enfermidade. Sua origem é atribuída a Rudolf Virchow que, no século XIX, aplicou o termo para as doenças compartilhadas entre os seres humanos e outros animais ${ }^{1}$. Atualmente, a Organização Mundial da Saúde define zoonose como: "Doenças ou infecções naturalmente transmissíveis entre animais vertebrados e seres humanos" 2.

Do total das doenças infecciosas existentes, $60 \%$ são classificadas como zoonoses. No século passado, das doenças infecciosas que emergiram ou reemergiram, mais de $75 \%$ são originárias de infecções em animais. Essa situação levou a Organização Mundial da Saúde, em 2006, a reconhecer a necessidade da criação de uma estratégia para a integração da infraestrutura e trabalho de setores das áreas da saúde, humana e animal, meio ambiente, agricultura e políticas sociais, para a resolução dos problemas relativos a essas doenças. Essa estratégia foi denominada estratégia de Saúde Única ("One Health"), colocando em pauta também a necessidade de uma rápida e consistente articulação e resposta de diversos setores em níveis regional, nacional e internacional ${ }^{3}$.

Um conjunto de ferramentas que está surgindo e que possui grande possibilidade de contribuir para essa demanda são as Tecnologias da Informação e Comunicação (TICs). Estas tecnologias podem ser entendidas como a articulação de competências humanas, metodológicas e tecnológicas subsidiadas por recursos computacionais. As TICs na área da saúde podem ser sistematizadas em três grupos: processamento, armazenamento e 
comunicação de dados. Dentre as numerosas aplicações dessas ferramentas estão: dispositivos móveis, identificação biométrica, telemedicina, certificações digitais, vídeomonitoramento, informatização de dados, softwares, dentre várias outras ${ }^{4}$.

Com o passar dos anos, a quantidade de dados vem crescendo cada vez mais. Nesse contexto, a informação assume um papel fundamental para subsidiar a elaboração, o planejamento e a tomada de decisões das políticas de saúde. Essa demanda levou o Ministério da Saúde à criação de uma Política Nacional de Informação e Informática em Saúde (PNIIS) que norteia as ações das TICs no Sistema Único de Saúde (SUS). A PNIIS tem como principal preocupação a estruturação do e-Gov e do e-Saúde, que é a informatização e a interoperabilidade da informação para todas as áreas do governo e da saúde por meio da gestão das TICs. Como principais aspectos para a sua implementação temos: a dificuldade na obtenção e no tratamento de dados, o grande número de sistemas de informação e a falta de comunicação entre eles, incluindo a dificuldade de diálogo entre os diferentes níveis de atenção, o financiamento insuficiência para o setor, a deficiência na formação e qualificação profissional e, por último, a necessidade de organização do país frente às ações e às estratégias internacionais no campo das TICs em saúde. A PNIIS, com a gestão das TICs no contexto do SUS, tem a possibilidade de aumentar o acesso, a segurança, a transparência, a velocidade e o alcance das informações sobre doenças, agravos e diversas situações de saúde em setores variados, como o Controle Social, a Atenção à Saúde, a Vigilância em Saúde, a Gestão do Trabalho e a Educação na Saúde e para a Gestão ${ }^{5}$.

Apesar da enorme repercussão que as doenças infecciosas emergentes e reemergentes vêm ocupando no mundo, ainda existe uma grande escassez de informações sobre o processo saúde-doença das zoonoses, tanto junto às populações, quanto pelos profissionais de saúde. Como agravante, os territórios de maior prevalência dessas enfermidades estão localizados em áreas longínquas, com poucos recursos e infraestrutura precária. Nessas condições, as TICs são estratégicas para o subsídio aos profissionais de saúde na atuação sobre esse tipo de agravo e suas especificidades, haja vista a possibilidade do aumento da velocidade e do alcance da informação, bem como a superação das barreiras geográficas. Assim posto, objetivou-se com esse trabalho, sistematizar informações sobre as Tecnologias da Informação e Comunicação em Saúde que contribuem no enfrentamento das zoonoses. 


\section{Métodos}

Este estudo pressupõe uma revisão de literatura narrativa, ou seja, um overview que não utiliza critérios explícitos e sistemáticos para a busca e análise crítica da literatura. $\mathrm{A}$ busca pelos estudos não precisa esgotar as fontes de informações. Não aplica estratégias de busca sofisticadas e exaustivas. A seleção dos estudos e a interpretação das informações podem estar sujeitas à subjetividade dos autores ${ }^{6}$.

A revisão da literatura narrativa ou tradicional, quando comparada à revisão sistemática, apresenta uma temática mais aberta; dificilmente parte de uma questão específica bem definida, não exigindo um protocolo rígido para sua confecção; a busca das fontes não é pré-determinada e específica, sendo frequentemente menos abrangente. A seleção dos artigos é arbitrária, provendo o autor de informações sujeitas a viés de seleção, com grande interferência da percepção subjetiva ${ }^{7}$.

Os critérios para o desenvolvimento do presente estudo iniciaram-se com a pesquisa dos Descritores em Ciências da Saúde (DeCS), mais adequados ao tema do estudo no site da Biblioteca Virtual da Saúde (BVS) (http://decs.bvs.br/), no qual foram selecionados os seguintes assuntos: "ANÁLISE ESPACIAL", "TELEMEDICINA" e "ZOONOSES". Também foram usados os operadores lógicos booleanos and, or e not, que auxiliam a definir as relações combinatórias entre os termos em uma pesquisa na obtenção dos resultados mais representativos na busca por meio de bancos de dados.

Os DeCS foram utilizados para realizar pesquisas nas bases de dados SCIELO (http://www.scielo.org/php/index.php) e LILACS (http://lilacs.bvsalud.org/). Posteriormente, utilizou-se os seguintes filtros para a pesquisa nas bases de dados: publicação dentro do período de 2014 a 2018, assegurando dessa forma a utilização de experiências recentes, e pesquisas cujo país de origem tenha sido o Brasil, devido ao interesse em trabalhos que envolvem o contexto do SUS.

Os critérios de inclusão dos estudos para serem selecionados na presente revisão de literatura consideraram o formato de artigo científico, que tenha sido desenvolvido com alguma(s) TICs em saúde e estudos que envolvessem o SUS, para que fossem abordadas apenas doenças de caráter zoonótico. Os critérios de exclusão foram definidos como sendo os estudos em formatos diferentes de artigos científicos, a utilização de instrumentos metodológicos que não fossem as TICs em saúde, além de contextos diferentes do SUS e que abordassem doenças não zoonóticas. 


\section{Resultados}

A busca dos descritores nas duas bases de dados, LILACS e SCIELO, obteve um total de 835 artigos científicos. A busca com o descritor "ANÁLISE ESPACIAL" recuperou um total de 607 documentos, o descritor "TELEMEDICINA" encontrou 70 documentos e o "ZOONOSES" encontrou 158 documentos. Posteriormente, os dados foram analisados pelos critérios de inclusão e exclusão pré-determinados, e se fez necessária a eliminação de documentos repetidos.

Ao fim da análise, foram selecionados 12 artigos científicos para a revisão bibliográfica, que foram divididos em três categorias, de acordo com a TIC utilizada, a saber: Teleducação e Telemedicina, com dois artigos científicos; Sistemas de Informações em Saúde, com seis artigos e Análise Espacial, com quatro artigos.

Dentre as zoonoses encontradas nos artigos, destacaram-se a tuberculose, em três artigos científicos, a hantavirose, a leishmaniose visceral e a febre maculosa, ambas em dois artigos, além da doença de Chagas e da toxoplasmose em um artigo, incluindo um estudo que foi desenvolvido com várias zoonoses. Sobre os locais dos estudos, a região Nordeste foi a que teve mais casos observados, com quatro artigos realizados em estados e munícipios a saber: Salvador (BA), Imperatriz (MA), Aracajú (SE) e o estado do Ceará, seguido pela região Norte e Sudeste, ambas com dois estudos: Barcarena (PA) e no estado do Pará, da primeira, e Belo Horizonte (MG) e Valinhos (SP), da segunda. Além disso, foram encontrados quatro estudos contemplando todo o território nacional. As informações encontram-se sistematizadas no Quadro 1 (Anexo).

\section{a) Teleducação e Telemedicina}

Para essa categoria, foram selecionados dois artigos científicos que abordaram experiências envolvendo as TICs em saúde pelos processos educativos no contexto do SUS. Ferramentas computacionais da Educação à Distância (EAD) subsidiaram atividades de Educação em Saúde e de Educação Permanente em Saúde.

Na primeira experiência, a teleducação foi utilizada para a prevenção de zoonoses, sendo realizada por meio de uma pesquisa-intervenção, que foi desenvolvida com a aplicação de formulários eletrônicos (pré e pós-testes) e com o desenvolvimento de um curso EAD. O estudo foi desenvolvido com professores e estudantes de escolas municipais do Distrito Sanitário Cabula/Beirú, em Salvador (BA). Este território é uma área em situação 
de vulnerabilidade social, sob condições socioeconômicas precárias, sem saneamento básico, escassez de políticas públicas, grande população de animais de rua, de internações e de notificações de zoonoses. Os resultados do pré-teste destacaram a presença de animais nas moradias da quase totalidade dos participantes, o desconhecimento sobre zoonoses, além do grande risco de infecção que elas apresentam, pois a maioria já tinha sofrido mordeduras de cães e gatos, picadas de carrapatos e insetos, contato com água empoçada ou de enchentes etc. Essas informações foram utilizadas para auxiliar na construção dos conteúdos do curso em EAD realizado por meio da Plataforma Moodle, da Universidade Federal da Bahia. Os resultados do pós-teste demonstraram uma boa eficiência na aprendizagem da maioria dos tópicos avaliados. 0 curso teve alta adesão por parte dos professores, que representaram a maioria dos cursistas. Entretanto, foi registrado um alto índice de evasão, que chegou a $60 \%$, por parte dos estudantes ${ }^{8}$.

A experiência de Educação Permanente em Saúde foi desenvolvida para avaliar os conhecimentos, as atitudes e as práticas preventivas a respeito da febre maculosa, frente às novas mudanças no programa de vigilância realizadas pelo Ministério da Saúde. Profissionais de saúde vinculados ao SUS, de todo o país, participaram da pesquisa com o auxílio de um formulário eletrônico (FORMSUS). Foram enviados 426 questionários a profissionais vinculados às áreas endêmicas e às áreas silenciosas, que participaram de um treinamento sobre as mudanças no programa. Dos questionários, apenas 112 foram respondidos $(26,3 \%)$, mas com uma boa representatividade do território nacional, envolvendo 52 munícipios de todas as regiões. 0 principal motivo para a resposta ao questionário pelo profissional era o fato da ocorrência de casos da doença, pois a maioria dos participantes era de profissionais das regiões Sudeste e Centro-Oeste, com curso superior e pós-graduação, atuantes na vigilância epidemiológica. Como destaque, a pesquisa avaliou que os profissionais estavam cientes das novas mudanças ocorridas no programa da febre maculosa, mas com alguns pontos críticos relacionados às ações de prevenção que aconteciam somente no momento da ocorrência de novos casos da doença, a precarização de vínculos e a rotatividade de profissionais, além do desconhecimento da doença por muitos profissionais das áreas endêmicas ${ }^{9}$.

\section{b) Sistemas de Informações em Saúde}

Nesta categoria, foram selecionados seis artigos científicos que foram desenvolvidos com a utilização de algum SIS integrado ao SUS, vinculado aos serviços de saúde nas 
esferas nacional, regional ou local. Dentre os SIS mais utilizados, o Sistema Nacional de Agravos de Notificação (SINAN) obteve destaque, sendo trabalhado de forma exclusiva em três pesquisas e em conjunto com outros sistemas de informações em outras duas. Já o Sistema de Informação sobre Mortalidade (SIM) foi utilizado de forma exclusiva em uma pesquisa.

No estado do Ceará, um estudo descreveu o perfil epidemiológico dos casos de Leishmaniose Visceral (LV), realizando uma análise a partir dos dados secundários disponibilizados pelo SINAN no período de 2007 a 2011. Esses dados apontaram que a LV é uma doença endêmica no estado, tendo sido notificados casos em $88 \%$ dos munícipios do Ceará (162/188), com uma média anual de 596,8 casos, o que corresponde a aproximadamente $15 \%$ dos casos do Brasil. A capital, Fortaleza, foi o município que registrou a maior quantidade de casos da doença no país, com exatos $5,6 \%$ dos casos nacionais durante o período estudado. A maioria dos indivíduos acometidos pela doença era de adultos que, em sua maioria, eram analfabetos e com ensino fundamental incompleto ${ }^{10}$.

A síndrome cardiopulmonar por hantavírus (SCPH) foi analisada em todo território nacional por meio de dados do SINAN para o período de 2007 a 2012. Dentre os principais resultados, 8.456 casos de SCPH foram notificados, com a confirmação de $9 \%$ destes (756/8.456), sendo 91,1\% deles comprovados pela prova laboratorial (689/756). As maiores incidências da doença foram nos estados do Mato Grosso, Santa Catarina e no Distrito Federal, sendo que o coeficiente de letalidade médio para o período foi de $38,2 \%$, e o maior número de óbitos aconteceu nos estados do Amazonas, do Mato Grosso e de São Paulo ${ }^{11}$.

0 último estudo que trabalhou exclusivamente com o SINAN também foi sobre a hantavirose. Nele, foram descritas a magnitude e a distribuição temporal de casos e óbitos da doença no Brasil de 2007 a 2015. Foram notificados 1.060 casos e ocorreram 410 óbitos durante esse período. A letalidade por hantavirose teve uma média de $39 \%$ dos casos, com óbitos atingindo em sua maioria o sexo feminino, a faixa etária de idosos e crianças, e principalmente as regiões Norte e Sul do país ${ }^{12}$.

Em dois estudos, o SINAN foi utilizado em conjunto com outros SIS. Um estudo avaliou as atividades de controle da LV em Belo Horizonte (MG), no período de 2007 a 2011, utilizando dados do SINAN e do Sistema de Informações de Controle de Zoonoses local. Verificou-se a adequação da estratégia de controle do reservatório canino, com aumento da cobertura de áreas priorizadas da população canina examinada e da eutanásia dos cães com testes positivos, gerando redução na prevalência da doença na população canina ${ }^{13}$. 0 
outro estudo foi desenvolvido com o SINAN e o Sistema Integrado de Informações Penitenciárias, tendo o objetivo de identificar a situação da tuberculose e sua variação temporal em pessoas privadas de liberdade (PPL) e na população brasileira durante o período de 2007 a 2012. A taxa de incidência da tuberculose foi de 25 a 30 vezes maior nas PPL do que na população geral ${ }^{14}$.

O Sistema de Informação sobre Mortalidade (SIM) foi utilizado na elaboração de um trabalho que versou sobre a identificação do perfil dos óbitos por tuberculose pulmonar em um município do nordeste brasileiro durante o período de 2005 a 2014 . 0 estudo foi composto por 37 notificações de óbito cuja causa básica foi tuberculose pulmonar. A faixa etária principal dos casos de óbito era de pessoas com idade média de 60 anos, com predomínio da forma pulmonar, sem menção de confirmação bacteriológica ou histológica (81\%), do sexo masculino (65\%) e de cor parda $(67 \%)^{15}$.

\section{c) Análise Espacial}

Para essa categoria, foram selecionados quatro artigos científicos que desenvolveram estudos com a utilização de aplicativos e softwares para o geoprocessamento a partir de dados provenientes de SIS ou de bancos de dados de serviços de saúde do SUS. Dentre os aplicativos e softwares utilizados nos estudos, estão o ArcGIS, o TerraView e o Google Earth.

O software ArcGIS foi utilizado em dois estudos. O primeiro deles analisou espacialmente a ocorrência de tuberculose nas populações indígenas e não indígenas no estado do Pará de 2005 a 2013. Para a análise espacial, foram obtidas as coordenadas geográficas dos munícipios pelo IBGE. Posteriormente, os dados foram processados no ArcGIS, versão 10.2. Encontraram-se diferenças marcantes na incidência de tuberculose entre a população indígena e não indígena, alcançando 7.812/100 mil habitantes e 118/100 mil habitantes, respectivamente, sendo possível identificar regiões com alto risco de adoecimento ${ }^{16}$. 0 segundo estudo analisou a distribuição da doença de Chagas relacionada às variáveis epidemiológicas, ambientais e demográficas no munícipio de Barcarena (PA) no período de 2007 a 2014. Foram utilizados dados epidemiológicos e demográficos da Secretaria Municipal de Saúde de Barcarena e imagens de satélites do Instituto Nacional de Pesquisas Espaciais. Os dados de desmatamento foram obtidos por classificação de imagens de satélites, utilizando rede neural artificial. Foi confirmada a dependência espacial dos casos da doença com os diferentes tipos de desmatamento identificados no município, 
bem como aglomerados de casos em áreas urbanas e rurais ${ }^{17}$.

Uma pesquisa sobre toxoplasmose em gestantes foi realizada com auxílio do software TerraView (versão 4.2.2). Desenvolveu-se um estudo ecológico com a perspectiva de analisar a distribuição espacial da prevalência de anticorpos anti-toxoplasma em gestantes residentes na cidade de Aracajú, Sergipe, no período de janeiro a dezembro de 2012. Os bairros e regiões foram caracterizados de acordo com o Sistema de Informação da Atenção Básica (SIAB) do munícipio. Os dados referentes às gestantes foram coletados no Centro de Especialidades Médicas que é referência nos testes para o pré-natal no SUS. Além do TerraView, foi utilizado o estimador de intensidade Kernel. A soroprevalência encontrada para IgG foi de $68,5 \%$ e para IgM, de $0,36 \%$. A prevalência de IgG subiu em associação com o aumento da idade nos bairros mais antigos da capital e entre as mulheres mais jovens, a maior prevalência foi nos bairros de periferia. Quanto ao anticorpo $\operatorname{lgM}$, a concentração espacial também foi mais elevada em bairros da periferia ${ }^{18}$.

O último estudo dessa seção trabalhou com o auxílio do Google Earth para analisar o padrão epidemiológico e espacial da febre maculosa no município de Valinhos (SP), relacionando aspectos espaciais com características ambientais e de infraestrutura. $A$ pesquisa utilizou todos os casos das doenças com confirmação laboratorial e com notificação no SINAN no período de 2001 até 2012. Os locais foram georreferenciados na base cartográfica, usando o Google Earth (coordenadas geográficas) com correção de acordo com o Sistema Geodésico Brasileiro. Utilizou-se o estimador Kernel para a análise da densidade de casos no mapa. O predomínio dos casos foi do sexo masculino (79,6\%), na faixa etária de 20 a 49 anos (49\%), atingindo uma letalidade de 42,9\%. As capivaras foram notificadas como principal hospedeiro primário de Amblyomma Cajennense, espécie identificada nas pesquisas acarológicas no local de estudo. Os locais prováveis de infecção estão localizados, em sua maioria, próximos às coleções hídricas, pastos sujos e mata ciliar degradada $^{19}$.

\section{Discussão}

Embora tenha havido uma redução considerável no número de mortes causadas nas últimas décadas no Brasil, as doenças infecciosas continuam sendo um sério problema de Saúde Pública. As zoonoses contribuem de forma importante para essa situação, com destaque para a tuberculose, a esquistossomose, a leptospirose, a leishmaniose, a doença de Chagas, a malária e a febre amarela, dentre outras ${ }^{20,21,22}$. No presente estudo, algumas 
dessas doenças foram contempladas, como a tuberculose, a leishmaniose e a doença de Chagas.

Muitos são os entraves para a pesquisa com as doenças infecciosas na área da Saúde Pública. Dentre eles, os pesquisadores destacaram o fato dos centros de pesquisa biomédica e de saúde estarem concentrados principalmente na região Sudeste do Brasil ${ }^{21}$. Apesar desta condição, os resultados mostraram que a maioria dos artigos científicos utilizados na revisão foram de pesquisas oriundas da região Nordeste, com quatro estudos, e também com importante participação da região Norte, que foi o local de outros dois.

Os estudos da categoria Teleducação e Telemedicina trouxeram intervenções envolvendo professores e estudantes em um trabalho e profissionais de saúde em outro. As intervenções no formato EAD possibilitam uma grande interação entre os participantes dessa modalidade de ensino, sendo uma importante estratégia para a articulação e a mobilização social no enfretamento das doenças ${ }^{23,24}$. Esses aspectos foram vistos na pesquisa-intervenção de Educação em Saúde sobre zoonoses, realizada com professores e estudantes de uma comunidade em vulnerabilidade social, mostrando a importância da articulação entre as TICs em saúde com a realidade, com a utilização de dados do pré-teste na construção do curso em EAD, bem como o papel fundamental da escola nesse contexto.

O questionário no formato eletrônico (FORMSUS) utilizado com os profissionais de saúde na pesquisa sobre o conhecimento, a atitude e as práticas sobre a febre maculosa, mostra a praticidade e o grande alcance das TICs, conseguindo realizar uma pesquisa com trabalhadores de todas as regiões do país. As atividades via web conseguem promover uma importante aproximação ao superar as distâncias entre as pessoas, favorecendo dessa forma a construção de redes de aprendizagem baseadas no processo de trabalho e refletindo, assim, a importância dessa tecnologia no contexto da EPS ${ }^{23}$.

Os resultados sobre a categoria SIS mostraram a sua relevância para a vigilância, o monitoramento e a atenção em saúde no contexto das zoonoses. As análises sobre os SIS apresentaram-se ainda fragmentadas, com pouco diálogo entre si e com sistemas de outras áreas, com a maioria dos estudos realizados com base em um único sistema. Foi a partir essa lógica que os SIS foram organizados, assim como destaca a autora "(...) é preciso entendê-los como um produto socialmente determinado" (p.13) ${ }^{25}$. Essa construção vem da evolução das práticas de saúde do Brasil, que se desenvolveram por intermédio de uma racionalidade fragmentada, estruturando programas e ações em separado para cada 
doença, que, na maioria, eram infecciosas e apenas em momento de surtos ${ }^{25}$.

Os artigos sobre Análise Espacial revelaram estudos que relacionaram dados epidemiológicos das zoonoses com características demográficas, ambientais e de infraestrutura dos territórios, com o auxílio de vários softwares, colaborando com a opinião que a análise espacial tem capacidade de manipular dados espaciais (como mapas) e relacioná-los com outros dados de interesse, como prevalência, incidência, indicadores socioeconômicos e demográficos ${ }^{24}$. Complementando a importância da análise espacial em eventos na área de saúde, o espaço geográfico deve ser tomado como indicativo das condições de vida da população que nele reside, facilitando a sistematização da complexidade da organização social sem precisar fragmentá-la em diferentes variáveis ${ }^{26}$.

Algumas pesquisas mostraram a relação existente entre as zoonoses e as condições socioeconômicas e ambientais do território. A pesquisa de anticorpos anti-toxoplasmose em gestantes de Aracajú (SE), expôs que a maioria das mulheres positivas moravam na periferia da cidade. Em outros três estudos, foi possível avaliar que as populações em situação de vulnerabilidade social estão mais arriscadas à contaminação e a uma maior prevalência da doença. A pesquisa-intervenção realizada com professores e estudantes em um distrito de Salvador, deixou evidente um grande número de fatores de riscos de infecção por zoonoses. Dois estudos sobre tuberculose demonstraram o quanto a população carcerária, em um, e a indígena, no outro, estão mais infectadas do que a população em geral. A pesquisa sobre febre maculosa mostrou que as características ambientais podem estar ligadas à cadeia epidemiológica da doença, como áreas desmatadas e pastos sujos. Esses resultados corroboram com a condição de que as zoonoses são a maioria dentre as doenças infecciosas relacionadas à pobreza. Interesses do sistema econômico potencializam cada vez mais o desequilíbrio ambiental, as desigualdades sociais e a desestruturação de políticas públicas sociais, de saneamento e de saúde, determinando assim que os países e as regiões mais pobres tenham uma maior prevalência de tais doenças $^{27,28}$.

Esses limites nas condições sociais e econômicas de um território em relação à utilização das TICs em saúde revelam a importância de se conhecer as potencialidades e os limites da realidade para a escolha e o correto uso de uma tecnologia. Para contextualizar essa situação, temos o rádio, que teve papel fundamental na difusão de informações sobre a prevenção e o controle de surtos e epidemias de doenças infecciosas em alguns países da África, haja vista o alto índice de analfabetismo e a não condição de acesso a tecnologias 
mais modernas, como a internet, computador e celular ${ }^{24}$.

Atualmente, as TICs são imprescindíveis para o acesso, a atualização, a segurança e a transparência da informação, como também para o cruzamento com bancos de dados, redes sociais, termos de busca na internet, noticiários locais, m-health, dentre outros, favorecendo assim o subsídio à tomada de decisão pela gestão em saúde ${ }^{24}$. Esses aspectos são de extrema relevância no contexto de emergência e de reemergência de doenças no cenário internacional. Portanto, a vigilância global é uma estratégia fundamental no novo Regulamento Sanitário Internacional, além da necessidade de desenvolvimento de redes globais de vigilância de doenças e de bioterrorismo. Essas redes fornecem pela primeira vez em escala global informações em tempo real sobre possíveis surtos e epidemias de doenças infecciosas, situação essa só alcançada por meio das TICs em saúde ${ }^{29}$.

\section{Conclusão}

As zoonoses continuam sendo um grave problema de Saúde Pública no país, tendo importante participação no perfil epidemiológico de morbimortalidade por doenças infecciosas. As doenças de caráter zoonótico demonstraram ainda ter relação direta com a degradação ambiental, com a precariedade das condições socioeconômicas, de moradia e de infraestrutura das populações mais carentes e de seus territórios. Somada a esses aspectos, a fragilização das políticas públicas referentes ao saneamento básico, à assistência social e à saúde pública intensificam ainda mais a prevalência e a incidência dessas doenças.

A busca por artigos científicos sobre a utilização das TICs no enfretamento às zoonoses ratificou a problemática da escassez de informações sobre essas doenças no contexto do SUS, resultando em poucos estudos encontrados. As experiências que tiveram destaque foram aquelas desenvolvidas com os SIS e os softwares para análise espacial, ficando as experiências voltadas à teleducação e à telemedicina com o número menor de artigos. Essa situação traz à tona a importante demanda pela realização de atividades sobre as zoonoses subsidiadas pelas TICs no contexto do Sistema Único de Saúde e norteadas pela PNIIS, principalmente na perspectiva da Educação Permanente e da Educação em Saúde. 


\section{Referências}

1. Schwalbe CW. Medicina veterinaria y salud pública. México: CRAT, 1968. 896p.

2. Organização Mundial de Saúde (OMS). Zoonoses. Disponível em: <http://www.who.int/topics/zoonoses/en/ >. Acesso em 07 de nov. de 2018.

3. Vallat B. Editorial: "One Health". Boletin OIE, n.1, p.1-2, 2013.

4. Schmeil MA. Saúde e Tecnologia da Informação e Comunicação. Fisioterapia e Movimento, v.26, n.3, p.477-478, 2013.

5. Brasil, Ministério da Saúde. Secretaria Executiva. Departamento de Monitoramento e Avaliação do SUS. Política Nacional de Informação e Informática em Saúde. Brasília - DF: Ministério da Saúde, 2016. 56p.

6. Mattos P de C. Tipos de revisão de literatura. UNESP. São Paulo, p. 2, 2015.

7. Cordeiro AM, et al. Revisão sistemática: uma revisão narrativa. Revista do Colégio Brasileiro de Cirurgiões, v. 34, n. 6, p. 428-431, 2007.

8. Seixas MM; Nery G; Souza EP; Franke CR; Bavia ME; Barrouin-Melo SM. Formação de educadores para promoção da saúde única em distrito sanitário de Salvador: estudo piloto. Revista Baiana de Saúde Pública, v. 41, n. 2, p. 490-507, 2017.

9. Oliveira SV, Caldas EP; Limongi JE, Gazeta GS. Avaliação dos conhecimentos e atitudes de prevenção sobre a febre maculosa entre os profissionais de saúde no Brasil. Journal Health Biological Science, v. 4, n.3, p.152-159, 2016.

10. Cavalcante IJM; Vale MR. Aspectos epidemiológicos da leishmaniose visceral (calazar) no Ceará no período de 2007 a 2011. Revista Brasileira de Epidemiologia, v. 17, n. 4, p. 911-924, 2014.

11. Oliveira SV; Fonseca LX; Barros e Silva PMR; Pereira SVC; Caldas EP. Análise do perfil epidemiológico da hantavirose no Brasil no período de 2007 a 2012. Revista de Patologia Tropical, v. 43, n.2, p.131-142. 2014.

12. Fonseca LX; Oliveira SV; Duarte EC. Magnitude e distribuição dos óbitos por hantavirose no Brasil, 2007-2015. Epidemiologia e Serviços de Saúde, v.27, n.2, p.111, 2018.

13. Morais MHF; Fiuza VOP; Araújo VEM; Meneses FC; Carneiro M. Avaliação das atividades de controle da leishmaniose visceral em Belo Horizonte, Minas Gerais, 2006-2011. Epidemiologia e Serviços de Saúde, v.24, n.3, p.485-496, 2015.

14. Sacramento DS; Gonçalves MJF. Situação da tuberculose em pessoas privadas de liberdade no período de 2007 a 2012. Revista de Enfermagem UFPE, v. 11, n. 1, p.140-151, 2017.

15. Gomes da Silva FB; Sodré MB; Santos FS; Costa ACPJ; Lobato JSM; Oliveira FJF; Santos Neto M. Perfil dos óbitos por tuberculose pulmonar em um município do Nordeste brasileiro durante o período de 2005-2014. Arquivos de Ciências da Saúde UNIPAR, v.21, n. 3, p. 147-153, 2017.

16. Paiva BL; Azevedo JQ; Nogueira LMV; Santos BO; Rodrigues ILA; Santos MNA. Distribuição espacial de tuberculose nas populações indígenas e não indígenas do estado do Pará, Brasil, 2005-2013. Escola Anna Nery, v.21, n.4, p.1-7, 2017.

17. Souza Júnior AS; Palácios VRCM; Miranda CS; Costa RJF; Catete CP; Chagasteles EJ; Pereira ALRR; Gonçalves NV. Análise espaço-temporal da doença de Chagas e seus fatores de risco ambientais e demográficos no município de Barcarena, Pará, Brasil. Revista Brasileira de Epidemiologia, v. 20, n.4, p.742-755, 2017.

18. Inagaki ADM; Cardoso NP; Lopes PJPL; Alves JAB; Mesquita JRF; Araújo KCGM; Katagre $S$. Análise espacial da prevalência de toxoplasmose em gestantes de 
Aracajú, Sergipe, Brasil. Revista Brasileira de Ginecologia Obstétrica, v.36, n.12, p.535-540, 2014.

19. Nasser JT; Lana RC; Silva CMS; Lourenço RW; Cunha e Silva DC; Donalísio MR. Urbanização da febre maculosa brasileira em município da região Sudeste:

epidemiologia e distribuição espacial. Revista Brasileira de Epidemiologia, v. 18, n,2, p.299-312, 2015.

20. Brasil, Núcleo de Comunicação, Secretaria de Vigilância em Saúde Ministério da Saúde. Situação Epidemiológica das Zoonoses de interesse à Saúde Pública. Boletim eletrônico epidemiológico, Brasília - DF: Ministério da Saúde, Ano 9, n.1, 2009. 17p.

21. Barreto ML; Teixeira MG; Bastos Fl; Ximenes RAA; Barata RB; Rodrigues LC. Successes and failures in the control of infectious diseases in Brazil: social and environmental context, policies, interventions, and research needs. The Lancet, v. 377, n. 9780, p.1877-1889, 2011.

22. Waldman EA; Sato APS. Trajetória das doenças infecciosas no Brasil nos últimos 50 anos: um contínuo desafio. Revista de Saúde Pública, v.50, n.68, p.1-18, 2016.

23. Santos MLR; Pinto IC; Riccio NCR; Sampaio J; Soares CLM. Tecnologias de EAD na perspectiva da EPS: Apontamentos acerca de possíveis aproximações. In: Mandarino ACS; Gallo E; Gomberg E. (Orgs). Informar e Educar em Saúde: análises e experiências. Salvador - BA: EDUFBA, Editora Fiocruz, Cap. 2, p.27-46, 2014.

24. Abbott PA; Barbosa SA. Editorial. Usando Tecnologia da Informação e Mobilização Social para Combater Doenças. Acta Paulista de Enfermagem, v.28, n. 1, p.3-5, 2015.

25. Moraes IHS. Informações em Saúde: da Prática Fragmentada ao Exercício da Cidadania. São Paulo/Rio de Janeiro: Hucitec, 1994. 172p.

26. Barata RB. Como e por que as desigualdades sociais fazem mal à saúde. Rio de Janeiro: Fiocruz, 2009. 120p

27. Navarro MBMA; Filgueiras ALL; Coelho H; Asensi MD; Lemos E; Sidoni M; Soares MS; Cardoso TAO. Doenças Emergentes e Reemergentes, Saúde e Ambiente. In: Minayo MCS, Miranda AC. (Orgs.). Saúde e Ambiente Sustentável: Estreitando Nós. Rio de Janeiro: Fiocruz. Parte I, cap.2, p.37-49, 2002.

28. Brasil, Ministério da Saúde. Secretaria de Vigilância em Saúde. Departamento de Análise de Situação em Saúde. Saúde Brasil 2013: uma análise da situação de saúde e das doenças transmissíveis relacionadas à pobreza. Brasília - DF: Ministério da Saúde, 2014. 384p.

29. Castillo-Salgado C. Trends and directions of global public health surveillance. Epidemiologic Reviews, v.32, n.1, p.93-109, 2010. 


\section{Anexos}

Quadro 1. Artigos selecionados com a busca nas bases de dados.

\begin{tabular}{|c|c|c|c|c|c|}
\hline Categoria & Autor(es) & Contexto & TIC & Doença & Local \\
\hline \multirow{2}{*}{$\begin{array}{l}\text { Teleducação } \\
\text { e } \\
\text { Telemedicina }\end{array}$} & $\begin{array}{l}\text { Seixas et al. } \\
\quad(2017)\end{array}$ & $\begin{array}{l}\text { Professores e } \\
\text { estudantes da } \\
\text { rede pública }\end{array}$ & Curso EAD & Zoonoses & $\begin{array}{l}\text { Salvador - } \\
\text { BA }\end{array}$ \\
\hline & $\begin{array}{l}\text { Oliveira et } \\
\text { al. (2016) }\end{array}$ & $\begin{array}{l}\text { Profissionais de } \\
\text { Saúde }\end{array}$ & $\begin{array}{l}\text { Formulário } \\
\text { eletrônico } \\
\text { (FORMSUS) }\end{array}$ & Febre Maculosa & Brasil \\
\hline \multirow{6}{*}{$\begin{array}{l}\text { Sistemas de } \\
\text { Informações } \\
\text { em Saúde }\end{array}$} & $\begin{array}{c}\text { Cavalcante } \\
\text { e Vale } \\
(2014)\end{array}$ & $\begin{array}{c}\text { Perfil } \\
\text { epidemiológico } \\
2007 \text { a } 2011\end{array}$ & SINAN & $\begin{array}{l}\text { Leishmaniose } \\
\text { Visceral }\end{array}$ & Ceará \\
\hline & $\begin{array}{l}\text { Oliveira et } \\
\text { al. (2014) }\end{array}$ & $\begin{array}{c}\text { Perfil } \\
\text { epidemiológico } \\
2007 \text { a } 2012\end{array}$ & SINAN & Hantavirose & Brasil \\
\hline & $\begin{array}{l}\text { Fonseca, } \\
\text { Oliveira e } \\
\text { Duarte } \\
(2018)\end{array}$ & $\begin{array}{c}\text { Perfil } \\
\text { epidemiológico } \\
2007 \text { a } 2015\end{array}$ & SINAN & Hantavirose & Brasil \\
\hline & $\begin{array}{l}\text { Morais et } \\
\text { al. (2015) }\end{array}$ & $\begin{array}{c}\text { Avaliar } \\
\text { atividades de } \\
\text { controle }\end{array}$ & SINAN e SICZ & $\begin{array}{l}\text { Leishmaniose } \\
\text { Visceral }\end{array}$ & $\begin{array}{c}\text { Belo } \\
\text { Horizonte - } \\
\text { MG }\end{array}$ \\
\hline & $\begin{array}{c}\text { Sacramento } \\
\text { e Gonçalves } \\
\text { (2017) }\end{array}$ & $\begin{array}{l}\text { Situação da } \\
\text { doença na } \\
\text { população em } \\
\text { geral e } \\
\text { carcerária }\end{array}$ & SINAN e SIIP & Tuberculose & Brasil \\
\hline & $\begin{array}{c}\text { Gomes da } \\
\text { Silva et al. } \\
(2017)\end{array}$ & $\begin{array}{l}\text { Perfil dos óbitos } \\
2005-2014\end{array}$ & SIM & Tuberculose & $\begin{array}{c}\text { Imperatriz - } \\
\text { MA }\end{array}$ \\
\hline \multirow{4}{*}{$\begin{array}{l}\text { Análise } \\
\text { Espacial }\end{array}$} & $\begin{array}{l}\text { Inakagi et } \\
\text { al. (2014) }\end{array}$ & $\begin{array}{l}\text { Distribuição } \\
\text { espacial da } \\
\text { prevalência de } \\
\text { anticorpos }\end{array}$ & TerraView & Toxoplasmose & Aracajú - SE \\
\hline & $\begin{array}{l}\text { Moraes et } \\
\text { al. (2015) }\end{array}$ & $\begin{array}{l}\text { Padrão espacial } \\
2001 \text { a } 2012\end{array}$ & Google Earth & Febre Maculosa & Valinhos - SP \\
\hline & $\begin{array}{l}\text { Paiva et al. } \\
\text { (2017) }\end{array}$ & $\begin{array}{c}\text { Identificar } \\
\text { espacialmente } \\
\text { nas populações } \\
\text { indígenas e não } \\
\text { indígenas }\end{array}$ & ArcGIS 10.2 & Tuberculose & Pará \\
\hline & $\begin{array}{c}\text { Souza } \\
\text { Júnior et al. } \\
(2017)\end{array}$ & $\begin{array}{l}\text { Distribuição em } \\
\text { relação às } \\
\text { variáveis }\end{array}$ & ArcGIS 10.2 & $\begin{array}{l}\text { Doença de } \\
\text { Chagas }\end{array}$ & $\begin{array}{c}\text { Barcarena - } \\
\text { PA }\end{array}$ \\
\hline
\end{tabular}




\begin{tabular}{|l|l|l|l|l|}
\hline & & $\begin{array}{c}\text { ambientais e } \\
\text { demográficas }\end{array}$ & & \\
\hline
\end{tabular}

Fonte: Autores 